\title{
Approximate calculation of the potential profile in a graphene-based device
}

\author{
P. Marconcini, M. Macucci \\ Dipartimento di Ingegneria dell'Informazione, \\ Università di Pisa, Via Girolamo Caruso 16, 56122 Pisa, Italy.
}

\begin{abstract}
The study of the transport and noise properties of graphene-based devices requires the computation of the potential profile as a function of the applied bias voltages. However, an exact solution for the potential profile involves a complete self-consistent treatment of the electrostatic and transport equations, which is computationally very expensive. Here, generalising the approach proposed by Das et al., the authors describe an approximate method that allows the evaluation of the potential profile by properly modifying, as a function of the bias voltages applied to the gates, the profile for a reference bias point, which is supposed to be known. The proposed approach is not very demanding from the computational point of view and can be useful for simulations aimed at the interpretation of experimental results or at device design.
\end{abstract}




\section{INTRODUCTION}

Since the end of the past century, a significant research effort has been spent on the proposal, analysis and fabrication of novel devices at the nanoscale, based on new materials, geometries, technological solutions or operating principles. For example, the fabrication of nanodevices based on III-V heterostructures, characterised by a high-mobility two-dimensional (2D) channel, has allowed to study transport and noise [1-7] in the ballistic regime. Moreover, devices based on new geometries and operating principles (see e.g. Ref. [8]) have been proposed to overcome the scaling limits of the traditional CMOS (Complementary Metal-Oxide-Semiconductor transistor) circuits.

In the last decade, particular attention has been devoted to electronics based on carbon allotropes and in particular on graphene [9-15]. Graphene is a 2D honeycomb lattice of carbon atoms, which was isolated from graphite in 2004 by Novoselov et al. [16]. It exhibits very interesting electric properties: unconfined graphene has an extremely high room-temperature mobility and, being only one-atom thick, allows a very effective electrostatic control by means of nearby gates. Therefore several electronic applications have been proposed for this material, ranging from radiofrequency devices, to electrochemical and light sensors, to digital transistors [12]. An obstacle to the use of graphene in digital electronics is represented by the absence of an energy gap, a problem which can be al least partially overcome with the introduction of lateral confinement and doping or of bilayer graphene, that exhibits a gap in the presence of an orthogonal electric field $[17,18]$. Another interesting aspect is that, in an envelope-function description, the transport equation of monolayer graphene is formally represented by the Dirac equation $[11,19]$, that is the same equation which describes the relativistic behaviour of massless fermions, and thus it is characterised by phenomena, such as Klein tunneling, which typically appear only at relativistic speeds.

A numerical study of the conductance and noise properties of graphene-based devices can be performed with different levels of approximation. For small devices, containing a limited number of atoms, an ab initio or tight-binding simulation [18] of the whole device is possible, whereas for larger graphene samples an envelope-function approach, based on the solution of the Dirac equation [20,21], is generally preferred, in order to reduce the computational times. A very efficient solution of the Dirac equation in graphene ribbons can be performed, for example, using a Fourier-based approach $[14,21]$ in the reciprocal space or, equivalently, 
a sinc-based method [22] in the direct space.

In all cases, an accurate simulation of graphene-based devices requires the self-consistent solution of the electrostatic and transport equations. Indeed, the solution of the Poisson equation yields the potential profile, which is required to compute the transport properties of the device. However, in its turn, the solution of the transport equation yields the wave function across the device and thus, in a mean-field approximation, the charge density due to mobile carriers. Therefore the two coupled equations should be solved iteratively until a self-consistent solution is achieved.

This procedure is computationally very expensive, and therefore in several cases, especially when a preliminary, coarse analysis of the device properties is needed, the use of a less accurate potential landscape, obtained with the procedure described in the following, can be preferable.

The method we propose is an extension of that described by Das et al. [23, 24], who consider the effect of a single biased gate on the Fermi energy (or, equivalently, on the potential) of a graphene sheet, without, however, explicitly providing the possibility to include other contributions to the potential profile.

In general, the potential profile in a graphene device derives from the combined action of several contributions: our approach allows the inclusion of the effect of a number of independently biased gates, as well as of a bare potential not deriving from capacitive couplings.

\section{DESCRIPTION OF THE METHOD}

Let us introduce a coordinate frame $x, y$ in the graphene plane, where we consider the electrostatic action of several gates (in Fig. 1, as an example, we represent a graphene device with two biasing gates).

In each point $(x, y)$ of the graphene sheet, we can express the potential energy $U(x, y)$ as the sum of a bare potential $U_{0}(x, y)$, which is supposed to be known, and of a variation $\Delta U(x, y)$ (with respect to $\left.U_{0}(x, y)\right)$ induced by the electrostatic action of the $\left(N_{G}\right.$, in general) biased gates:

$$
U(x, y)=U_{0}(x, y)+\Delta U(x, y)
$$

In general, if $U_{0}(x, y)$ is known when the gates are biased at the voltages $V_{G i_{0}}$, the term $\Delta U(x, y)$ represents the contribution to $U(x, y)$ of the variations of the gate voltages with 


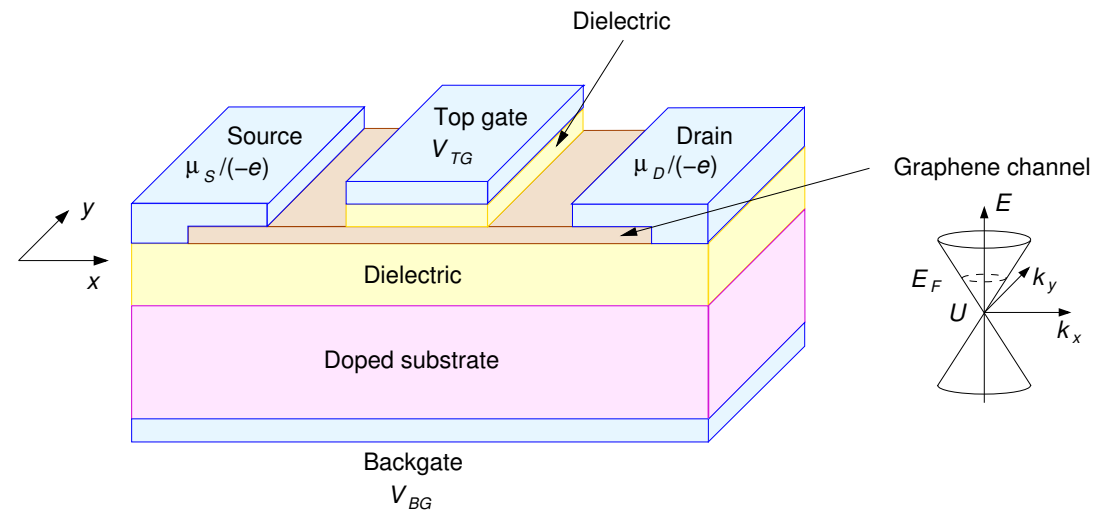

FIG. 1. Sketch of a graphene transistor with two biasing gates: a backgate and a top gate. On the right, we represent the energy dispersion relation of unconfined graphene near each of the Dirac points where the conduction band touches the valence band, as a function of the wave vector $\left(k_{x}, k_{y}\right)$ measured from the Dirac point.

respect to $V_{G i_{0}}$.

The term $U_{0}(x, y)$ includes the effect of the graphene sheet doping, of the charged impurities located both inside the dielectric separating the graphene sheet from the gates and inside the sheet itself, and in general all the contributions not deriving from the gate voltages included in the term $\Delta U(x, y)$.

The quantity $U_{0}(x, y)$ is often known from experimental data or obtained in an approximate form through simplified analytical or semi-analytical approaches.

For example, if the doping profile is known, its effect on the bare potential $U_{0}$ can be obtained with a semiclassical approach. Moreover, the contribution to $U_{0}$ of charged impurities randomly located outside the graphene sheet can be taken into consideration with techniques, which are available in the literature, to approximate the screened disordered potential they induce on the graphene sheet, for example modelling the contributions of the impurities with Gaussian functions with randomly distributed amplitudes [25].

In detail, in Ref. [26], Koschny and Schweitzer have shown that a disordered potential given by the sum of Gaussian contributions:

$$
U(\vec{r})=\frac{2 W}{\eta \sqrt{2 \pi n_{i m p}}} \sum_{i} \alpha_{i} e^{-\left|\vec{r}-\vec{R}_{i}\right|^{2} / \eta^{2}},
$$

(where $\eta / \sqrt{2}$ is the variance of the Gaussians, $W$ is the strength of the disordered potential, $\alpha_{i}$ is a random variable uniformly distributed between -1 and 1 and the random positions $\vec{R}_{i}$ 
are uniformly distributed, with concentration $n_{i m p}$, over the considered area) has a Gaussian autocorrelation function:

$$
\left\langle U\left(\vec{r}_{i}\right) U\left(\vec{r}_{j}\right)\right\rangle \approx \frac{W^{2}}{3} e^{-\left|\vec{r}_{i}-\vec{r}_{j}\right|^{2} /\left(2 \eta^{2}\right)}
$$

In the case of disordered graphene, Adam et al. [27] have provided relations between the Gaussian autocorrelation function and the physical parameters of the problem: the impurity concentration $n_{i m p}$, the distance $d$ of the impurities from the graphene plane, the effective permittivity $\varepsilon_{r}$ and the Fermi energy $E_{F}=\left(\hbar v_{F}\right) k_{F}$ (where $\hbar$ is the reduced Planck constant, $v_{F}$ is the Fermi velocity of graphene and $k_{F}$ is the Fermi wave vector). They have expressed the autocorrelation function as:

$$
\left\langle U\left(\vec{r}_{i}\right) U\left(\vec{r}_{j}\right)\right\rangle \approx \frac{K_{0}\left(\hbar v_{f}\right)^{2}}{2 \pi \eta^{2}} e^{-\left|\vec{r}_{i}-\vec{r}_{j}\right|^{2} /\left(2 \eta^{2}\right)}
$$

(where the dimensionless parameter $K_{0}$ is a function of the disorder strength, range and concentration, whereas the correlation length $\eta$ represents an estimate of the typical size of the electron-hole puddles in the graphene sample). Using the definitions:

$$
r_{s}=\frac{e^{2}}{4 \pi \varepsilon_{0} \varepsilon_{r}\left(\hbar v_{F}\right)}, \quad z=4 k_{F} d, \quad E_{1}[x]=\int_{x}^{\infty} \frac{e^{-t}}{t} d t
$$

(where $e$ is the modulus of the electron charge, and the effective relative permittivity $\varepsilon_{r}$ is given by the average between the relative permittivities of the media above and below the graphene sheet), they have found that

$$
K_{0}=\frac{1}{4 r_{s}^{2}}\left(\frac{D_{0}}{C_{0}}\right)^{2}, \quad \eta=\frac{1}{\sqrt{n_{i m p}}} \frac{D_{0}}{4 \pi r_{s}^{2}} \frac{1}{\left(C_{0}\right)^{3 / 2}},
$$

with

$$
\begin{aligned}
& C_{0}(z)=-1+\frac{4 E_{1}[z]}{\left(2+\pi r_{s}\right)^{2}}+\frac{2 e^{-z} r_{s}}{1+2 r_{s}}+\left(1+2 z r_{s}\right) e^{2 z r_{s}}\left(E_{1}\left[2 z r_{s}\right]-E_{1}\left[z\left(1+2 r_{s}\right)\right]\right) \\
& D_{0}(z)=1-\frac{8 r_{s} z E_{1}[z]}{\left(2+\pi r_{s}\right)^{2}}+\frac{8 e^{-z} r_{s}}{\left(2+\pi r_{s}\right)^{2}}-\frac{2 e^{-z} r_{s}}{1+2 r_{s}}-2 z r_{s} e^{2 z r_{s}}\left(E_{1}\left[2 z r_{s}\right]-E_{1}\left[z\left(1+2 r_{s}\right)\right]\right) .
\end{aligned}
$$

Substituting the actual physical parameters into these relations, the autocorrelation function of the potential, and thus the values required to simulate the contribution of the charged impurities through a superposition of Gaussian functions, can be obtained.

Once the potential profile $U_{0}(x, y)$ is known, the presence of gate bias voltages

$$
V_{G i}=V_{G i_{0}}+\Delta V_{G i}
$$


(differing by the quantities $\Delta V_{G i}$ from the values $V_{G i_{0}}$ for which $U_{0}(x, y)$ has been determined) alters the value of the graphene potential profile $U(x, y)$ by an amount $\Delta U(x, y)$.

In order to estimate $\Delta U(x, y)$, we have to take into consideration the charge that the gate voltages induce in the graphene sheet.

The variations $\Delta V_{G i}$ of the gate voltages with respect to the reference voltages $V_{G i_{0}}$ cause in each point $(x, y)$ of the graphene layer a variation $\Delta \rho(x, y)$ of the charge density $\rho(x, y)$ with respect to the value $\rho_{0}(x, y)$ which would otherwise be present. Therefore we can express the $2 \mathrm{D}$ charge density in graphene as

$$
\rho(x, y)=\rho_{0}(x, y)+\Delta \rho(x, y)
$$

using a naming of the variables analogous to that used in Eq. (1).

We assume to work in quasi-equilibrium conditions, that is, with $\mu_{S} \approx \mu_{D} \approx E_{F}$ (where $\mu_{S}$ and $\mu_{D}$ are the electrochemical potentials of the source and of the drain, respectively). If the potential is slowly varying [28], we can also assume that the energy dispersion relations are locally shifted by the value of the potential energy $U(x, y)$. Therefore we can approximate the local density of states (which would in principle require the knowledge of the wave function all over the graphene sample and thus the complete self-consistent solution of the electrostatic and transport equations) with the density of states evaluated in $E-U(x, y)$ (where $E$ is the energy).

The total charge density can be easily obtained by summing up the density of the holes in the valence band, multiplied by the hole charge $e$, and the density of the electrons in the conduction band, multiplied by the electron charge $-e$. Under the approximation of slowly varying potential, these densities are obtained with an energy integration of the density of states (evaluated in $E-U$ ) multiplied by the occupation function, which in the case of electrons corresponds to the Fermi-Dirac function $f\left(E-E_{F}\right)$ (where $f(x)=$ $1 /\left(1+\exp \left(x /\left(k_{B} T\right)\right)\right)$, with $k_{B}$ the Boltzmann constant and $T$ the absolute temperature), and in the case of holes to $1-f\left(E-E_{F}\right)$.

Therefore in each point $(x, y)$ the total charge density can be approximately written as:

$$
\begin{aligned}
\rho=\rho_{0}+\Delta \rho & =e \int_{-\infty}^{U_{0}+\Delta U} \operatorname{DOS}\left(E-U_{0}-\Delta U\right)\left[1-f\left(E-E_{F}\right)\right] d E \\
& -e \int_{U_{0}+\Delta U}^{\infty} \operatorname{DOS}\left(E-U_{0}-\Delta U\right) f\left(E-E_{F}\right) d E
\end{aligned}
$$


where, because of the shift of the energy bands, the energy level where the conduction band touches the valence band corresponds to the potential energy $U(x, y)$ (see Fig. 1). In this equation, we have expressed $U(x, y)$ and $\rho(x, y)$ using Eqs. (1) and (9), and we have omitted the dependence on $x$ and $y$, for the sake of simplicity.

Notice that when the hypothesis of quasi-equilibrium is not verified, the method can be extended modifying Eq. (10) as follows (as long as no inelastic scattering is assumed in the device):

$$
\begin{aligned}
\rho=\rho_{0}+\Delta \rho & =e \int_{-\infty}^{U_{0}+\Delta U} \operatorname{DOS}\left(E-U_{0}-\Delta U\right)\left[1-f\left(E-E_{F_{D}}\right)\right] d E \\
& -e \int_{U_{0}+\Delta U}^{\infty} \operatorname{DOS}\left(E-U_{0}-\Delta U\right) f\left(E-E_{F_{S}}\right) d E
\end{aligned}
$$

where $E_{F_{S}}$ and $E_{F_{D}}$ are the Fermi energies at the source and drain contacts. Indeed, in the absence of inelastic scattering, electrons and holes keep the energy distribution and the Fermi energy of their emitting contact (the source and the drain, respectively) throughout the device.

We can obtain Eq. (10) also observing that charge neutrality is achieved when there is a $\pi$ electron for each carbon atom, that is, when the valence band is completely full, whereas the conductance band is empty: in that condition the charge of the electrons is exactly neutralised by the charge of the nuclei of the carbon atoms. Therefore, $\rho(x, y)$ can be obtained computing the charge (per unit area) of all the occupied electron states and subtracting the electron charge corresponding to a full valence band. Separating the total electron charge into those deriving from the valence and from the conduction bands, the previous expression is recovered:

$$
\begin{aligned}
\rho & =-e \int_{-\infty}^{+\infty} \operatorname{DOS}(E-U) f\left(E-E_{F}\right) d E-\left[-e \int_{-\infty}^{U} \operatorname{DOS}(E-U) d E\right] \\
& =-e \int_{-\infty}^{U} \operatorname{DOS}(E-U) f\left(E-E_{F}\right) d E-e \int_{U}^{+\infty} \operatorname{DOS}(E-U) f\left(E-E_{F}\right) d E \\
& +e \int_{-\infty}^{U} \operatorname{DOS}(E-U) d E \\
& =e \int_{-\infty}^{U} \operatorname{DOS}(E-U)\left[1-f\left(E-E_{F}\right)\right] d E-e \int_{U}^{\infty} \operatorname{DOS}(E-U) f\left(E-E_{F}\right) d E,
\end{aligned}
$$

where $U=U_{0}+\Delta U$ and we have omitted again the spatial dependence.

This expression can be further simplified if we are simulating a device operating at low temperature. In such a case, the Fermi-Dirac function can be approximated with the unit 
step function $u$ :

$$
f\left(E-E_{F}\right)=u\left(E_{F}-E\right)
$$

(where $u$ is equal to 1 if its argument is positive and to 0 if it is negative). Then, Eq. (10) can be rewritten as:

$$
\begin{aligned}
\rho=\rho_{0}+\Delta \rho & =\left[e \int_{E_{F}}^{U_{0}+\Delta U} \operatorname{DOS}\left(E-U_{0}-\Delta U\right) d E\right] u\left(U_{0}+\Delta U-E_{F}\right) \\
& +\left[-e \int_{U_{0}+\Delta U}^{E_{F}} \operatorname{DOS}\left(E-U_{0}-\Delta U\right) d E\right] u\left(E_{F}-U_{0}-\Delta U\right)
\end{aligned}
$$

(where the step-function factors $u$ which multiply the two terms between square brackets express the fact that $\rho$ is equal to the first term if $E_{F}<U$ and to the second term if $\left.E_{F}>U\right)$. More briefly, we have that:

$$
\rho_{0}+\Delta \rho=e \int_{E_{F}}^{U_{0}+\Delta U} \operatorname{DOS}\left(E-U_{0}-\Delta U\right) d E .
$$

In each point $(x, y)$ of the graphene layer, the charge density $\rho_{0}(x, y)$ corresponding to the absence of gate voltage shifts $\Delta V_{G i}$ can be easily computed from the knowledge of $U_{0}(x, y)$ setting $\Delta U$ to zero in the previous expression:

$$
\rho_{0}=e \int_{E_{F}}^{U_{0}} \operatorname{DOS}\left(E-U_{0}\right) d E .
$$

Instead, when the gate voltages are shifted by $\Delta V_{G i}$, they electrostatically induce a further charge density $\Delta \rho(x, y)$ in the graphene sheet, which is obtained through a variation of the number of states that are occupied by electrons at the considered Fermi energy (established by the potential at the contacts). Since the density of states in graphene is finite, this $\Delta \rho(x, y)$ is at the origin of the finite variation $\Delta U(x, y)$ of the potential: the appearance of $\Delta U(x, y)$ causes the shift (with respect to the Fermi energy) of the local energy dispersion relations (and thus the change in the local density of states) which generates the required variation of the number of occupied electron states.

In order to write a simplified electrostatic relation between the voltage and charge variations, we introduce the geometrical capacitances per unit area $C_{G i}(x, y)$ (which are assumed to be known, once the device geometry has been established) between each gate and the generic point $(x, y)$ of the graphene layer.

For each point $(x, y)$ we can consider an equivalent circuit relating the voltage variations, in which the gates are coupled to the graphene region by means of capacitors with capacitance $C_{G i}(x, y)$, with a voltage $\Delta V_{G i}$ on the plate corresponding to the gate and a voltage 


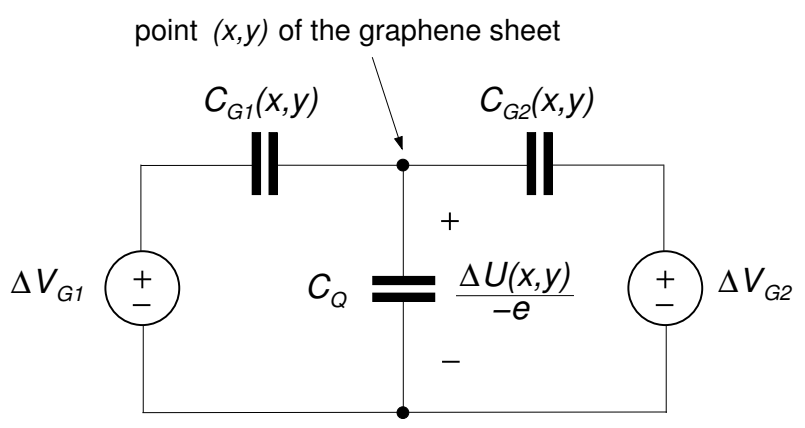

FIG. 2. Equivalent circuit that relates the variations of the gate voltages to the change of the potential on the graphene plane. For example, here we consider two biasing gates.

$\Delta U /(-e)$ on the plate corresponding to the graphene elementary area. As a consequence, the overall variation of charge per unit area $\Delta \rho(x, y)$ is the sum of the charges on the graphene plates of the $N_{G}$ capacitors (which represent the charges induced in the graphene sheet by the voltages $\left.\Delta V_{G i}\right)$, according to

$$
\Delta \rho=\sum_{i} C_{G i}\left(\frac{\Delta U}{-e}-\Delta V_{G i}\right)
$$

(we divide $\Delta U$ by $-e$ in order to convert it into the corresponding potential).

If only small variations were considered, the equivalent circuit could be completed with the graphene quantum capacitance $C_{Q}$ (between the graphene flake and the reference node) [29], which relates $\Delta \rho$ and $\left(\Delta\left(E_{F}-U\right)\right) /(-e)$ (where $E_{F}-U$ describes the position of the Fermi energy with respect to the energy bands) through the density of states $\operatorname{DOS}(E-U)$ of graphene (see Fig. 2).

More in detail, the quantum capacitance is defined as:

$$
C_{Q}=\frac{\Delta \rho}{\left(\Delta\left(E_{F}-U\right)\right) /(-e)}=\frac{-e \Delta n}{\left(\Delta\left(E_{F}-U\right)\right) /(-e)}=e^{2} \frac{\Delta n}{\Delta\left(E_{F}-U\right)}
$$

(where $n$ is the electron concentration in the graphene flake and in our case $\Delta\left(E_{F}-U\right)=$ $-\Delta U)$. From a circuit point of view, enforcing the condition of charge neutrality in the node which represents the graphene sheet, we have that

$$
C_{Q}\left(\frac{\Delta U}{-e}\right)+\sum_{i} C_{G i}\left(\frac{\Delta U}{-e}-\Delta V_{G i}\right)=0 .
$$

From this relation, it follows that

$$
\Delta \rho=-C_{Q}\left(\frac{\Delta U}{-e}\right)=\sum_{i} C_{G i}\left(\frac{\Delta U}{-e}-\Delta V_{G i}\right),
$$


which corresponds to Eq. (17).

Since (in the hypotheses of quasi-equilibrium and low temperature)

$$
n=\int_{-\infty}^{E_{F}} \operatorname{DOS}(E-U) d E=\int_{-\infty}^{E_{F}-U} \operatorname{DOS}(\alpha) d \alpha
$$

(with $\alpha=E-U$ ), if small variations are considered the quantum capacitance can be expressed as

$$
C_{Q} \approx e^{2} \frac{\partial n}{\partial\left(E_{F}-U\right)}=e^{2} \operatorname{DOS}\left(E_{F}-U\right) .
$$

In general, the quantum capacitance written in this form depends on the relative position of the potential energy with respect to the Fermi energy, and thus is not constant over significant variations of the gate voltages.

In our calculation we do not explicitly consider the quantum capacitance, but we instead describe the relation (governed by the density of states of graphene) between $\Delta \rho$ and $\Delta U$ through Eq. (15), which connects the total quantities $\rho$ and $U$, including their variations. Even large variations of the gate voltages are allowed, since Eq. (10) relates the total quantities (and thus does not involve differential parameters) and in Eq. (17) only the geometrical capacitances, which are constant (i.e. not depending on the particular values of the energies) parameters, appear.

Therefore, in order to compute the variation $\Delta U(x, y)$ resulting from the application of the voltages $\Delta V_{G i}$, we just have to solve the system made up of Eqs. (15) and (17):

$$
\left\{\begin{aligned}
\rho_{0}+\Delta \rho & =e \int_{E_{F}}^{U_{0}+\Delta U} \operatorname{DOS}\left(E-U_{0}-\Delta U\right) d E \\
\Delta \rho & =\sum_{i} C_{G i}\left(\frac{\Delta U}{-e}-\Delta V_{G i}\right)
\end{aligned}\right.
$$

for each point $(x, y)$ of the graphene layer (we recall that $\rho_{0}, \Delta \rho, U_{0}, \Delta U$, and $C_{G i}$ depend on $x$ and $y$ ), where $U_{0}$ is known and $\rho_{0}$ is given by Eq. (16). This corresponds to solving the equation (substituting $\Delta \rho$ from the second equation into the first equation):

$$
\rho_{0}+\sum_{i} C_{G i}\left(\frac{\Delta U}{-e}-\Delta V_{G i}\right)=e \int_{E_{F}}^{U_{0}+\Delta U} \operatorname{DOS}\left(E-U_{0}-\Delta U\right) d E .
$$

In the case of unconfined monolayer graphene (which is also a good approximation for large ribbons) and for the low energies for which the envelope-function approximation is valid, the density of states is given by [11]

$$
\operatorname{DOS}(E)=\frac{2|E|}{\pi\left(\hbar v_{F}\right)^{2}}
$$


(for smaller ribbons this expression should be replaced with that derived in Ref. [29]).

Therefore, Eq. (15) becomes:

$$
\rho_{0}+\Delta \rho=\frac{2 e}{\pi\left(\hbar v_{F}\right)^{2}} \int_{E_{F}}^{U_{0}+\Delta U}\left|E-U_{0}-\Delta U\right| d E .
$$

Since for $E_{F}<U_{0}+\Delta U$ the argument of the modulus is negative in the overall integration range, whereas for $E_{F}>U_{0}+\Delta U$ it is positive, this relation can be rewritten as:

$$
\rho_{0}+\Delta \rho=\operatorname{sign}\left(E_{F}-U_{0}-\Delta U\right) \frac{2 e}{\pi\left(\hbar v_{F}\right)^{2}} \int_{E_{F}}^{U_{0}+\Delta U}\left(E-U_{0}-\Delta U\right) d E .
$$

The integral is easily solvable performing the change of variable $\epsilon=E-U_{0}-\Delta U$ :

$$
\begin{aligned}
\rho_{0}+\Delta \rho & =\operatorname{sign}\left(E_{F}-U_{0}-\Delta U\right) \frac{2 e}{\pi\left(\hbar v_{F}\right)^{2}} \int_{E_{F}-U_{0}-\Delta U}^{0} \epsilon d \epsilon \\
& =\operatorname{sign}\left(U_{0}+\Delta U-E_{F}\right) \frac{e}{\pi\left(\hbar v_{F}\right)^{2}}\left(U_{0}+\Delta U-E_{F}\right)^{2} .
\end{aligned}
$$

Therefore the system (23) becomes:

$$
\left\{\begin{aligned}
\rho_{0}+\Delta \rho & =\operatorname{sign}\left(U_{0}+\Delta U-E_{F}\right) \frac{e}{\pi\left(\hbar v_{F}\right)^{2}}\left(U_{0}+\Delta U-E_{F}\right)^{2} \\
\Delta \rho & =\sum_{i} C_{G i}\left(\frac{\Delta U}{-e}-\Delta V_{G i}\right)
\end{aligned}\right.
$$

where $U_{0}$ is known and $\rho_{0}$ is given by

$$
\rho_{0}=\operatorname{sign}\left(U_{0}-E_{F}\right) \frac{e}{\pi\left(\hbar v_{F}\right)^{2}}\left(U_{0}-E_{F}\right)^{2} .
$$

The resulting equation (24) becomes

$$
\rho_{0}+\sum_{i} C_{G i}\left(\frac{\Delta U}{-e}-\Delta V_{G i}\right)=\operatorname{sign}\left(U_{0}+\Delta U-E_{F}\right) \frac{e}{\pi\left(\hbar v_{F}\right)^{2}}\left(U_{0}+\Delta U-E_{F}\right)^{2} .
$$

In order to find the value of $\Delta U$, we have to solve this equation twice, once for each of the two possible signs of $U_{0}+\Delta U-E_{F}$.

If $U_{0}+\Delta U-E_{F} \geq 0$ the equation becomes

$$
\begin{aligned}
{\left[\frac{e}{\pi\left(\hbar v_{F}\right)^{2}}\right](\Delta U)^{2}+} & {\left[\frac{2 e}{\pi\left(\hbar v_{F}\right)^{2}}\left(U_{0}-E_{F}\right)+\sum_{i} \frac{C_{G i}}{e}\right] \Delta U } \\
+ & {\left[\frac{e}{\pi\left(\hbar v_{F}\right)^{2}}\left(U_{0}-E_{F}\right)^{2}-\rho_{0}+\sum_{i} C_{G i} \Delta V_{G i}\right]=0 }
\end{aligned}
$$






FIG. 3. Approximate representation, as a function of $\Delta U$, of the two sides of Eq. (34). The meaning of $\alpha, \beta$, and $\chi$ is explained in the text. The actual position of the two curves depends on the values of these quantities. The dashed lines represent the axes of the reference frame. The solution $\Delta U$ of Eq. (34) is given by the abscissa of the intersection point between the two curves.

while for $U_{0}+\Delta U-E_{F}<0$ it becomes

$$
\begin{aligned}
{\left[\frac{e}{\pi\left(\hbar v_{F}\right)^{2}}\right](\Delta U)^{2}+} & {\left[\frac{2 e}{\pi\left(\hbar v_{F}\right)^{2}}\left(U_{0}-E_{F}\right)-\sum_{i} \frac{C_{G i}}{e}\right] \Delta U } \\
+ & {\left[\frac{e}{\pi\left(\hbar v_{F}\right)^{2}}\left(U_{0}-E_{F}\right)^{2}+\rho_{0}-\sum_{i} C_{G i} \Delta V_{G i}\right]=0 . }
\end{aligned}
$$

In both cases, it is a second degree equation in $\Delta U: a(\Delta U)^{2}+b(\Delta U)+c=0$. If the discriminant $b^{2}-4 a c$ is negative, no solution exists for the corresponding choice of the sign of $U_{0}+\Delta U-E_{F}$. Instead, if the discriminant is positive, we have to check if one of the two possible solutions $\Delta U=\left(-b \pm \sqrt{b^{2}-4 a c}\right) /(2 a)$ is consistent with the hypothesis that has been made for the sign of $U_{0}+\Delta U-E_{F}$.

As one could expect from a physical point of view, the problem has a single solution. This can be deduced rewriting Eq. (31) as:

$$
\left(\rho_{0}-\sum_{i} C_{G i} V_{G i}\right)-\left(\sum_{i} \frac{C_{G i}}{e}\right) \Delta U=\operatorname{sign}\left(\left(U_{0}-E_{F}\right)+\Delta U\right) \frac{e}{\pi\left(\hbar v_{F}\right)^{2}}\left(\left(U_{0}-E_{F}\right)+\Delta U\right)^{2} .
$$

If we plot the two sides of this equation as a function of $\Delta U$, we observe that the left-hand side is a straight line with a negative slope and thus a monotonically decreasing function, whereas the right-hand side is a monotonically increasing curve (consisting of a parabola with positive curvature for $\Delta U \geq E_{F}-U_{0}$ and of a parabola with negative curvature for 


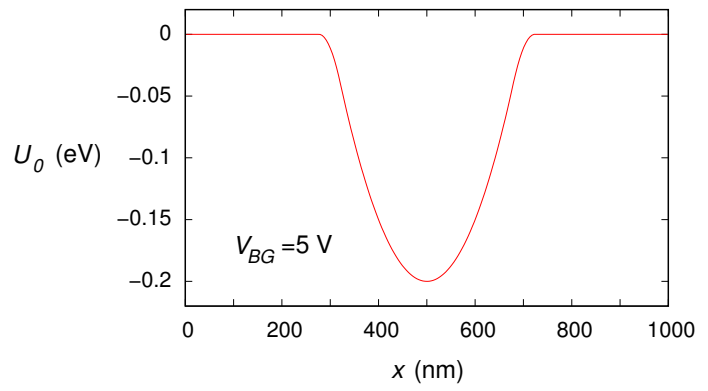

FIG. 4. Longitudinal behaviour of the (transversally constant) bare potential $U_{0}$ that we have assumed to know for $V_{B G}=5 \mathrm{~V}$. In detail, defining $\tilde{x}=x-500 \mathrm{~nm}$, we have taken: $U_{0}=0$ for $\tilde{x} \leq-x_{1}$ and for $\tilde{x} \geq x_{1}, U_{0}=-C\left(\tilde{x}+x_{1}\right)^{2}$ for $-x_{1} \leq \tilde{x} \leq-x_{2}, U_{0}=A \tilde{x}^{2}-B$ for $-x_{2} \leq \tilde{x} \leq x_{2}$ and $U_{0}=-C\left(\tilde{x}-x_{1}\right)^{2}$ for $x_{2} \leq \tilde{x} \leq x_{1}$, with $x_{1}=100 \sqrt{5} \mathrm{~nm}, x_{2}=80 \sqrt{5} \mathrm{~nm}$, $A=5 \times 10^{-6} \mathrm{eV} / \mathrm{nm}^{2}, B=200 \mathrm{meV}, C=20 \times 10^{-6} \mathrm{eV} / \mathrm{nm}^{2}$.

$\Delta U<E_{F}-U_{0}$ ). Therefore only one intersection between the two curves (and thus one solution of the equation) exists (see Fig. 3, where we have used the following shorthands: $\alpha=\rho_{0}-\sum_{i}\left(C_{G i} V_{G i}\right), \beta=\sum_{i}\left(C_{G i} / e\right)$, and $\left.\chi=E_{F}-U_{0}\right)$.

In the form we have just presented, the method is valid at low temperature and in quasiequilibrium conditions $\left(\mu_{S} \approx \mu_{D}\right)$. As previously stated, if the temperature is not low (or the device is not in equilibrium conditions and no inelastic scattering is present), it is instead necessary to solve the system of Eq. (10) and of Eq. (17) (or of Eq. (11) and of Eq. (17), respectively), using Eq. (25) for the graphene density of states. However, in this case a numerical, iterative procedure will be necessary to solve the system.

\section{NUMERICAL RESULTS AND COMPARISON WITH PREVIOUS APPROACHES}

We have first considered a graphene sheet in the presence of a backgate, assuming the bare potential $U_{0}$ to be known for a backgate voltage $V_{B G}$ equal to $V_{B G_{0}}=5 \mathrm{~V}$. We have considered $U_{0}$ transversally constant and varying along the longitudinal direction $x$ with the behaviour represented in Fig. 4.

We have assumed the backgate to be coupled to the graphene sheet by a constant capacitance per unit area equal to $C_{B G}=0.1151 \mathrm{mF} / \mathrm{m}^{2}$ (corresponding to the presence of a $300 \mathrm{~nm}$ thick layer of $\mathrm{SiO}_{2}$ between the gate and the graphene flake). We have considered 

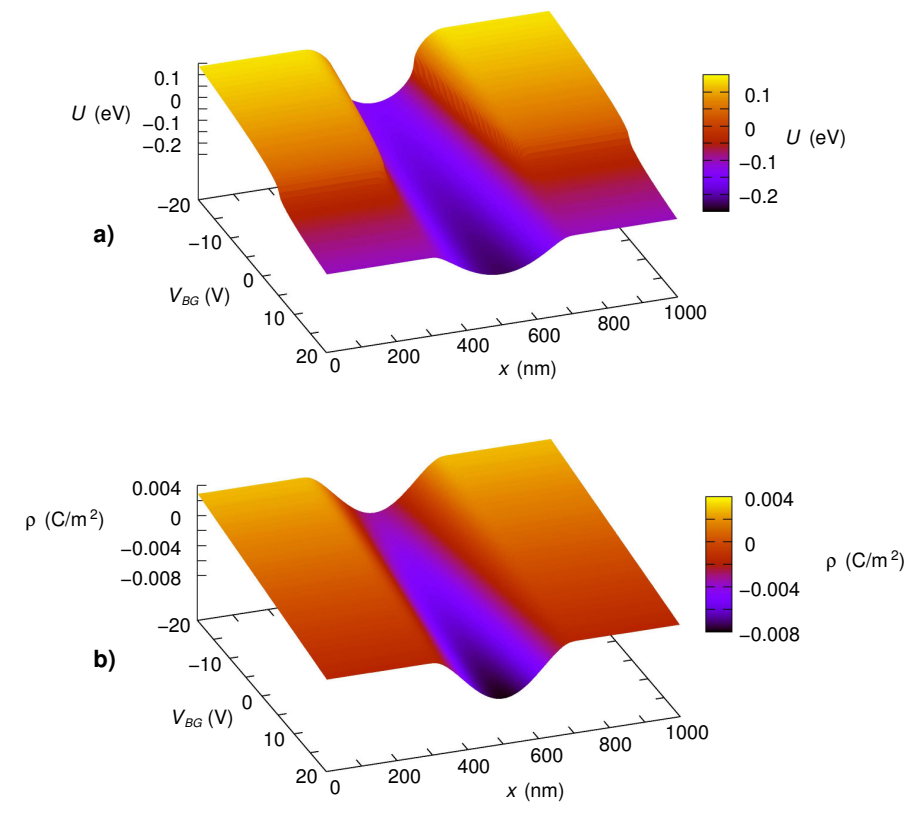

FIG. 5. Cross-section of $U$ (panel (a)) and of $\rho$ (panel (b)) along the longitudinal direction, in the middle of the flake, represented as a function of $x$ and of the voltage $V_{B G}$ on the backgate. The bare potential for $V_{B G}=5 \mathrm{~V}$ has the profile shown in Fig. 4. The capacitance coupling the backgate and the graphene flake is $C_{B G}=0.1151 \mathrm{mF} / \mathrm{m}^{2}$.

$E_{F}=0$ and we have varied the voltage $V_{B G}$ applied to the backgate between -20 and $20 \mathrm{~V}$. In Fig. 5, we report the cross-section of the resulting $U$ (panel (a)) and $\rho$ (panel (b)) along the longitudinal direction, in the middle of the flake, as a function of $x$ and of $V_{B G}$. Note that, as long as in Eq. (17) $\Delta U /(-e)$ is much less than $\Delta V_{B G}$, the behaviour of $\rho$ as a function of $V_{B G}$ is nearly linear.

Then, we have included disorder (in addition to the potential with the profile represented in Fig. 4) into $U_{0}$. The potential disorder has been approximately represented with a sum of Gaussian functions randomly distributed over the flake (see Eq. (2)), with concentration $n_{i m p}=5 \times 10^{11} \mathrm{~cm}^{-2}$, random amplitude uniformly distributed between -120 and $120 \mathrm{meV}$ and half-width at half-maximum equal to $5 \mathrm{~nm}$. These values have been obtained from Eqs. (2)-(7), considering $E_{F}=50 \mathrm{meV}, d=1 \mathrm{~nm}$ and $\varepsilon_{r}=2.5$. In Fig. 6, we represent a cross-section of the resulting $U_{0}$ (for $V_{B G}=5 \mathrm{~V}$ ) along the longitudinal direction, in the middle of the graphene sheet. In Fig. 7, instead, we report the cross-section of $U$ (panel (a)) and of $\rho$ (panel (b)) in the middle of the flake as a function of $x$ and of $V_{B G}$, computed 




FIG. 6. Cross-section, along the longitudinal direction, in the middle of the graphene sheet, of the bare potential $U_{0}$ (for $V_{B G}=5 \mathrm{~V}$ ) obtained adding some disorder to the potential represented in Fig. 4.

assuming again $E_{F}=0$ and $C_{B G}=0.1151 \mathrm{mF} / \mathrm{m}^{2}$.

As a further example, we have added the effect of a probe, biased with a voltage $V_{P}$, located at a certain distance from the graphene layer. The projection of the probe position on the graphene flake is $\left(x_{P}, y_{P}\right)$, with $x_{P}=200 \mathrm{~nm}$ and $y_{P}$ in the middle of the sheet. The coupling between the probe and the generic point $(x, y)$ of graphene is represented by a capacitance per unit area with a Lorentzian dependence $C_{T G}=C_{T G_{\max }} /\left(1+\left(d / d_{0}\right)^{2}\right)$ (with $C_{T G_{\max }}=0.1 \mathrm{mF} / \mathrm{m}^{2}$ and $\left.d_{0}=50 \mathrm{~nm}\right)$ on the distance $d$ between $(x, y)$ and $\left(x_{P}, y_{P}\right)$. In this example, we assume that at the reference backgate potential $V_{B G_{0}}$ and for $V_{P}=0$ the bare potential has a profile identical to that represented in Fig. 4 . We consider $E_{F}=0$ and $C_{B G}=0.1151 \mathrm{mF} / \mathrm{m}^{2}$. If we vary $V_{P}$ between -20 and $20 \mathrm{~V}$ keeping $V_{B G}$ constant at $V_{B G_{0}}$, in the middle of the flake we obtain the values of $U$ and of $\rho$ represented in Fig. 8 (panel (a) and panel (b), respectively) as a function of $x$ and of $V_{P}$.

Finally, let us compare our equation with that previously used in the literature in a few simpler situations. In particular, let us consider a device with a single gate and let us assume $\rho_{0}=0, U_{0}=0$, and $E_{F}=0$. In this case, Eq. (31) becomes

$$
C_{G}\left(\frac{\Delta U}{-e}-\Delta V_{G}\right)=\operatorname{sign}(\Delta U) \frac{e}{\pi\left(\hbar v_{F}\right)^{2}}(\Delta U)^{2}
$$

which can be rewritten as:

$$
e \Delta V_{G}=-\operatorname{sign}(\Delta U) \frac{e^{2}}{\pi\left(\hbar v_{F}\right)^{2} C_{G}}(\Delta U)^{2}-\Delta U
$$

Since shifting the energy dispersion relations by $\Delta U$ while keeping the Fermi energy constant 

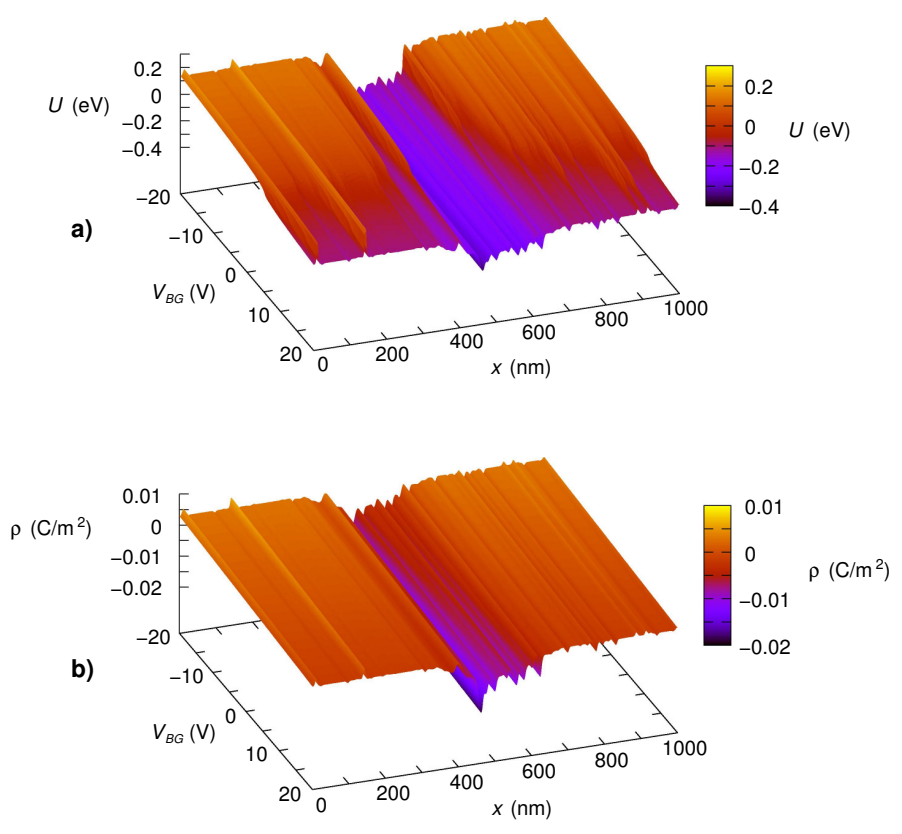

FIG. 7. Cross-section of $U$ (panel (a)) and of $\rho$ (panel (b)) along the longitudinal direction, in the middle of the flake, represented as a function of $x$ and of the voltage $V_{B G}$ on the backgate. The bare potential for $V_{B G}=5 \mathrm{~V}$ has been obtained adding some disorder to the potential represented in Fig. 4; its cross section in the middle of the graphene sheet is shown in Fig. 6. The capacitance coupling the backgate and the graphene flake is $C_{B G}=0.1151 \mathrm{mF} / \mathrm{m}^{2}$.

at 0 is equivalent to keeping the energy bands constant while shifting the Fermi energy by $-\Delta U$, this corresponds to the expression used by Das et al. in Refs. [23, 24].

Under the further hypothesis that $\left|e \Delta V_{G}\right|>>|\Delta U|$ (which corresponds to neglecting $\Delta U /(-e)$, with respect to $\Delta V_{G}$, in the electrostatic equation (17)) the term $-\Delta U$ can be disregarded, and the equation becomes:

$$
\Delta V_{G}=\operatorname{sign}\left(\Delta V_{G}\right)\left|\Delta V_{G}\right|=-\operatorname{sign}(\Delta U) \frac{e}{\pi\left(\hbar v_{F}\right)^{2} C_{G}}(\Delta U)^{2} .
$$

Since a voltage shift $\Delta V_{G}>0(<0)$ applied to the gate induces a charge variation $\Delta \rho<0$ $(>0)$ on the unit area of the graphene sheet, which corresponds to a shift $\Delta U<0$ (> $0)$ of the energy bands and thus to a potential shift $\Delta U /(-e)>0(<0)$, we have that $\operatorname{sign}(\Delta U /(-e))=\operatorname{sign}\left(\Delta V_{G}\right)$. Therefore we conclude that

$$
\left|\Delta V_{G}\right|=\frac{e^{3}}{\pi\left(\hbar v_{F}\right)^{2} C_{G}}\left(\frac{\Delta U}{-e}\right)^{2}
$$



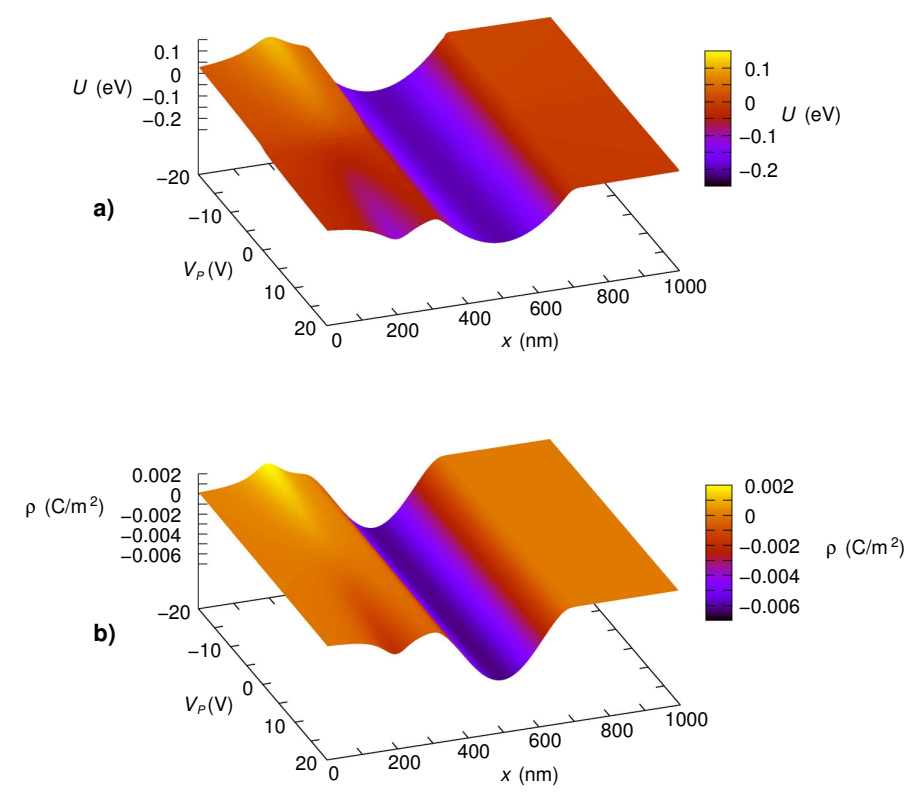

FIG. 8. Cross-section of $U$ (panel (a)) and of $\rho$ (panel (b)) along the longitudinal direction, in the middle of the flake, represented as a function of $x$ and of the voltage $V_{P}$ on the probe. The bare potential at the reference backgate voltage (which is kept constant) and for $V_{P}=0$ is assumed to be the same as that represented in Fig. 4. We assume the capacitive coupling between the probe and the points of the graphene flake to have a Lorentzian spatial dependence, whereas $C_{B G}=0.1151 \mathrm{mF} / \mathrm{m}^{2}$.

and thus

$$
\left|\frac{\Delta U}{-e}\right|=\sqrt{\frac{\pi\left(\hbar v_{F}\right)^{2} C_{G}}{e^{3}}} \sqrt{\left|\Delta V_{G}\right|} .
$$

This is in agreement with the approximate relation reported in Ref. [30], where the gate is separated from the graphene layer by a silicon oxide layer with a thickness $t_{o x}=300 \mathrm{~nm}$. In that case, the value of the gate geometrical capacitance is $C_{G}=\varepsilon_{0} \varepsilon_{r} / t_{o x} \approx 0.1151 \mathrm{mF} / \mathrm{m}^{2}$ and thus it is possible to make an estimation of the effect of the gate voltage on the potential of the graphene layer using the relation $|\Delta U /(-e)|=\sqrt{\left|\Delta V_{G}\right|} / 36.6$, where both $|\Delta U /(-e)|$ and $\left|\Delta V_{G}\right|$ are expressed in volt (if $|\Delta U /(-e)|$ is expressed in $\mathrm{mV}$ the result is analogous to that reported in Ref. [30]). 


\section{CONCLUSION}

We have presented a method to compute the effect of the voltages applied to a number of bias gates on the potential in a graphene sheet, taking into account the presence of a non-zero bare potential profile evaluated for a reference bias condition (and in this way generalising existing approaches [23, 24]).

The obtained potential landscape (which includes the screening effect in an approximate way) can be used as an input for a non-self-consistent transport simulation (using proper numerical methods, such as those described in Refs. [20, 21]), in such a way as to avoid the more exact but computationally expensive self-consistent solution of the electrostatic and transport equations. This makes a quick evaluation of the transport characteristics of graphene devices as a function of the applied gate voltages possible, and therefore is especially useful during the design and optimisation phase, when a short turnaround time is needed for simulations run to understand the effect of changes in the device parameters.

It is to be noted that this method can be applied also in the presence of a scanning probe, which can be treated as a particular type of gate, and, in general, for arbitrary gate geometries.

[1] Van Wees, B. J., van Houten, H., Beenakker, C. W. J., et al: 'Quantized conductance of point contacts in a two-dimensional electron gas', Phys. Rev. Lett. 1988, 60, (9), pp. 848-850

[2] Wharam, D. A., Thornton, T. J., Newbury, R., et al: 'One-dimensional transport and the quantisation of the ballistic resistance', J. Phys. C: Solid State Phys., 1988, 21, (8), pp. L209L214

[3] Marconcini, P., Macucci, M., Iannaccone, G., Pellegrini, B., Marola, G.: 'Analysis of shot noise suppression in mesoscopic cavities in a magnetic field', Europhys Lett., 2006, 73, (4), pp. $574-580$

[4] Whitney, R. S., Marconcini, P., Macucci, M.: 'Huge conductance peak caused by symmetry in double quantum dots', Phys. Rev. Lett., 2009, 102, (18), pp. 186802-1-4

[5] Marconcini, P., Macucci, M., Iannaccone, G., Pellegrini, B.: 'Quantum analysis of shot noise suppression in a series of tunnel barriers', Phys. Rev. B, 2009, 79, (24), pp. 241307(R)-1-4 
[6] Marconcini, P., Macucci, M., Logoteta, D., Totaro, M.: 'Is the regime with shot noise suppression by a factor $1 / 3$ achievable in semiconductor devices with mesoscopic dimensions?', Fluct. Noise Lett., 2012, 11, (1), pp. 1240012-1-12

[7] Marconcini, P., Totaro, M., Basso, G., Macucci, M.: 'Effect of potential fluctuations on shot noise suppression in mesoscopic cavities', AIP Advances, 2013, 3, (6), pp. 062131-1-13

[8] D’Amico, P., Marconcini, P., Fiori, G., Iannaccone, G.: 'Engineering Interband Tunneling in Nanowires With Diamond Cubic or Zincblende Crystalline Structure Based on Atomistic Modeling', IEEE Trans. Nanotechnol., 2013, 12, (5), pp. 839-842

[9] Avouris, Ph., Chen, Z., Perebeinos, V.: 'Carbon-based electronics', Nature Nanotech., 2007, $2,(10)$, pp. $605-615$

[10] Saito, R., Dresselhaus, G., Dresselhaus, M. S.: 'Physical Properties of Carbon Nanotube' (Imperial College Press, 1998)

[11] Castro Neto, A. H., Guinea, F., Peres, N. M. R., Novoselov, K. S., Geim, A. K.: 'The electronic properties of graphene', Rev. Mod. Phys., 2009, 81, (1), pp. 109-162

[12] Schwierz, F.: 'Graphene transistors', Nature Nanotech., 2010, 5, (7), pp. 487-496

[13] Marconcini, P., Macucci, M.: 'A novel choice of the graphene unit vectors, useful in zonefolding computations', Carbon, 2007, 45, (5), pp. 1018-1024

[14] Fagotti, M., Bonati, C., Logoteta, D., Marconcini, P., Macucci, M.: 'Armchair graphene nanoribbons: $\mathcal{P} \mathcal{T}$-symmetry breaking and exceptional points without dissipation', Phys. Rev. B, 2011, 83, (24), pp. 241406(R)-1-4

[15] Connolly, M. R., Puddy, R. K., Logoteta, D., et al: 'Unraveling Quantum Hall Breakdown in Bilayer Graphene with Scanning Gate Microscopy', Nano Lett., 2012, 12, (11), pp. 5448-5454

[16] Novoselov, K. S., Geim, A. K., Morozov, S. V., et al.: 'Electric Field Effect in Atomically Thin Carbon Films', Science, 2004, 306, (5696), pp. 666-669

[17] Iannaccone, G., Fiori, G., Macucci, M., et al: 'Perspectives of graphene nanoelectronics: probing technological options with modeling', Proceedings of the International Electron Devices Meeting 2009 (IEDM 2009), Baltimore, Maryland (USA), December 2009, pp. 245-248, DOI: 10.1109/IEDM.2009.5424376

[18] Marconcini, P., Cresti, A., Triozon, F., et al: 'Atomistic Boron-Doped Graphene Field-Effect Transistors: A Route toward Unipolar Characteristics', ACS Nano, 2012, 6, (9), pp. 7942-7947

[19] Marconcini, P., Macucci, M.: 'The $\vec{k} \cdot \vec{p}$ method and its application to graphene, carbon 
nanotubes and graphene nanoribbons: the Dirac equation', La Rivista del Nuovo Cimento, 2011, 34, (8-9), pp. 489-584, DOI: 10.1393/ncr/i2011-10068-1

[20] Tworzydło, J., Groth, C. W., Beenakker, C. W. J.: 'Finite difference method for transport properties of massless Dirac fermions', Phys. Rev. B, 2008, 78, (23), pp. 235438-1-10

[21] Marconcini, P., Macucci, M.: 'Symmetry-dependent transport behavior of graphene double dots', J. Appl. Phys., 2013, 114, (16), pp. 163708-1-6

[22] Marconcini, P., Logoteta, D., Macucci, M.: 'Sinc-based method for an efficient solution in the direct space of quantum wave equations with periodic boundary conditions', J. Appl. Phys., 2013, 114, (17), pp. 173707-1-10

[23] Das, A., Chakraborty, B., Piscanec, S., Pisana, S., Sood, A. K., Ferrari, A. C.: 'Phonon renormalization in doped bilayer graphene', Phys. Rev. B, 2009, 79, (15), pp. 155417-1-7

[24] Das, A., Pisana, S., Chakraborty, B., et al.: 'Monitoring dopants by Raman scattering in an electrochemically top-gated graphene transistor' Nature Nanotech., 2008, 3, (4), pp. 210-215

[25] Rycerz, A., Tworzydło, J., Beenakker, C. W. J.: 'Anomalously large conductance fluctuations in weakly disordered graphene', Europhys. Lett., 2007, 79, (5), 57003

[26] Koschny, Th., Schweitzer, L.: 'Levitation of the quantum Hall extended states in the $B \rightarrow 0$ limit', Phys. Rev. B, 2004, 70, (16), 165301

[27] Adam, S., Cho, S., Fuhrer, M. S., Das Sarma, S.: 'Density Inhomogeneity Driven Percolation Metal-Insulator Transition and Dimensional Crossover in Graphene Nanoribbons', Phys. Rev. Lett., 2008, 101, (4), pp. 046404-1-4

[28] Ashcroft, N. W., Mermin, N. D.: 'Solid State Physics' (Brooks/Cole, 1976), pp. 340-341

[29] Fang, T., Konar, A., Xing, H., Jena D.: 'Carrier statistics and quantum capacitance of graphene sheets and ribbons', Appl. Phys. Lett., 2007, 91, (9), pp. 092109-1-3

[30] Gorbachev, R. V., Mayorov, A. S., Savchenko, A. K., Horsell, D. W., Guinea, F.: 'Conductance of p-n-p Graphene Structures with “Air-Bridge” Top Gates', Nano Lett., 2008, 8, (7), pp. 19951999 